\title{
On Computing and Drawing Maxmin-Height Covering Triangulation *
}

\author{
Binhai Zhu and Xiaotie Deng \\ Dept. of Computer Science, City University of Hong Kong, Kowloon, Hong Kong \\ SAR, China. \{bhz, deng\}@cs.cityu.edu.hk.
}

\section{Introduction}

Given a simple polygon $P$, a covering triangulation is another triangulation over the vertices of $P$ and some inner Steiner points (see Fig 1 for a covering triangulation generated by our heuristic). In other words, when computing a covering triangulation one is only allowed to add Steiner points in the interior of $P$. This problem is originally from mesh smoothing: one is not happy with the mesh over a specific region (say $P$ ) and would like to re-triangulate that region. Certainly, adding Steiner points on the boundary of $P$ would destroy the neighboring part of $P$ and would result in further changes of the mesh.

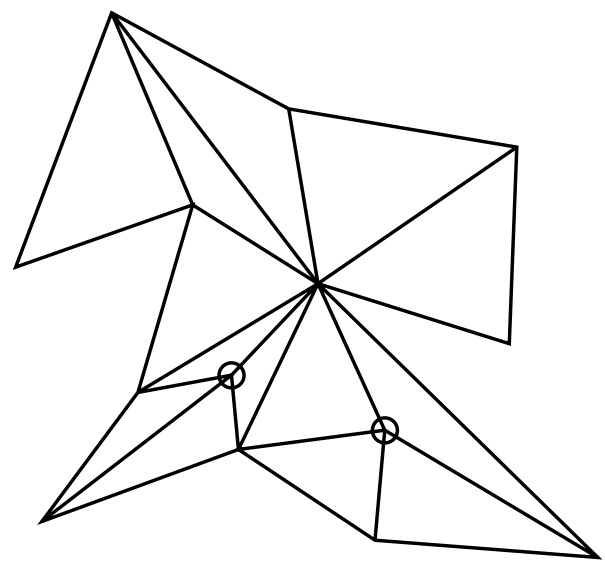

Fig. 1. A covering triangulation built by our heuristic algorithm, Steiner points are round.

When computing a covering triangulation of $P$, we usually want to make sure that the triangles have certain good property (and in fact this results in aesthetically beautiful covering triangulation). Among these properties, minmax angle, maxmin angle have received much attention. Mitchell obtained good approximation algorithms for optimizing both of these qualities [Mit94. Mit97. However,

\footnotetext{
* This work is partially supported by the research grant No. 7000743 from City University of Hong Kong and UGC grant CityU1049/98E.
} 
for another important covering triangulation, namingly the one maximizing the minimum height, very little is known.

In this report we briefly mention some facts we already know about the maxmin height covering triangulation. Then we introduce a heuristic for generating a covering triangulation which seems to have good empirical results.

\section{Results}

First of all, we briefly introduce something we know about maxmin height covering triangulation. Because of the space constraint, we do not illustrate any of the proofs.

Theorem 1. Given a simple polygon, the smaller of the minimum vertex/edge distance and the minimum edge length is the upper bound of the optimal height in the maxmin height covering triangulation.

Theorem 2. Given a simple polygon, the minimum length stable diagonal is the upper bound of the optimal height in the maxmin height covering triangulation.

Here the vertex/edge distance between an edge $e$ and a vertex $v$ of $P$ is the vertical distance between $v$ and $v_{1}$ where $v v_{1}$ is vertical to $e$ and $v_{1}$ lies in the interior of $e$; moreover, $v v_{1}$ does not intersect $P$. A stable diagonal of $P$ is the diagonal of $P$ which appears in any triangulation of $P$. Clearly a convex polygon has no stable diagonal and a non-convex 4-gon has exactly one stable diagonal.

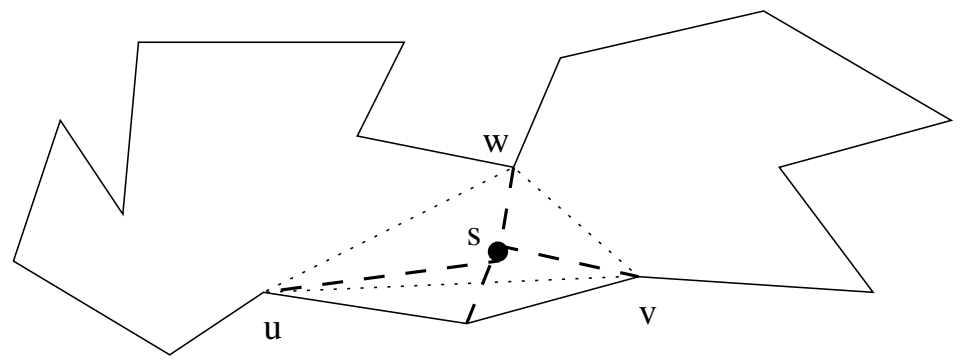

Fig. 2. An illustration of the heuristic algorithm.

Unfortunately the above facts do not immediately lead to any good approximate (or exact) solution. Nevertheless, we present a very simple heuristic to compute and draw a high quality covering triangulation. The idea is to try to cut an ear off at each stage. We note that when cutting an ear off (i.e., by adding a diagonal $u v$ ) this diagonal might lead to very small height in the ear. If this happens, then we will add an interior Steiner point $s$ to increase the local triangle heights and to decompose the polygon into two pieces (to the left of the wedge 
usw and to the right of the wedge $v s w$ in Fig 2) which allow us to recursively apply this heuristic. (There are several such cases and here we just illustrate one of them, because of the space constraint.) However, we cannot prove formally that this heuristic lead to a good approximation solution as after each step the upper bound we illustrate in Theorem 1 and 2 changes. We run our heuristic over many manually input polygons, the empirical results seem to be promising (see Fig 1 for an example). Clearly this heuristic runs in $O\left(n^{2}\right)$ time theoretically if we employ a linear time algorithm to slice off an ear EET93.

Closing Remarks. We note that if the Steiner points are allowed to be on the boundary of $P$, then constant factor approximate solution exists [BDE95]. We raise several problems to conclude this report. (1) Can we design a good approximate algorithm for this problem? (2) What can we say theoretically about our simple heuristic?

\section{References}

[BDE95] M. Bern, D. Dobkin and D. Eppstein. Triangulating polygons without large angles. Intl. J. Comput. Geom. and Appl., 5:171-192, 1995.

[Mit94] S. Mitchell. Finding a covering triangulation whose maximum angle is provably small. Proc. 17th Australian Computer Science Conf. pages 55-64, 1994.

[Mit97] S. Mitchell. Approximating the maxmin-angle covering triangulation. Comput. Geom. Theo. and Appl., 7:93-111, 1997.

[EET93] H. ElGindy, H. Everett and G. Toussaint. Slicing an ear in linear time. Patt. Recog. Lett., 14:719-722, 1993. 